\title{
Research on Tourism Image Perception of Tang Paradise
}

\author{
Yue $\operatorname{Sun}^{1, *}$ \\ ${ }^{1}$ Tourism Management, School of Economics and Management, Beijing Jiaotong University \\ ${ }^{*}$ Corresponding author. Email: 20120727@bjtu.edu.cn
}

\begin{abstract}
In the Internet age, it is scientific to use the analysis of online texts to explore tourists' image perception of tourist attractions. This study selects Tang Paradise as a case site, uses ROST Content 6.0 software to analyze the web texts obtained from the Ctrip.com and Mafengwo website,obtains the tourism image of Tang Paradise from the perspective of tourists' perception. The results show: Tourists have more perceptions of tourist attractions in Tang Paradise, among which the perception of human resources is the deepest; semantic network analysis diagram, including "Tang Paradise - park - ticket" and other three important relationship chain;the overall perception of tourists is positive, but in terms of tourist consumption and tourism infrastructure, negative emotions account for a relatively high proportion . Based on the results of this analysis, relevant suggestions are made for the improvement of the tourist image of Tang Paradise.
\end{abstract}

Keywords: Network text analysis, Tourist attractions, Image perception, Tang paradise.

\section{INTRODUCTION}

With the rapid economic development, people's trips continue to increase, and their perception of the image of tourist attractions has also deepened. Relying on the characteristics of the wide coverage and fast propagation speed of the Internet platform, tourists can quickly learn about the tourist resources, infrastructure and other aspects of the tourist attractions. The tourist attractions management can know the real feelings of the tourists after the visit, and it is also beneficial to potential tourists make travel and optimize the image of tourist attractions.

Based on the "cognitive-emotion" model and communication theory, this paper uses online texts to study tourists' perception of the image of Tang Paradise. To some extent, it makes up the shortcoming of using fixed questions and options in traditional questionnaire survey. Using content analysis to transform non-systematic symbolic content into quantitative data can more intuitively show the true perception of tourists.

\section{LITERATURE REVIEW}

The image of tourist destinations was proposed by Hunt (1971), who defined people's impression of non-residential places as the image of tourist destinations [1]. Choi et al. (2007) used the network information of the tourism platform to analyze the tourist destination image of Macau [2]. Greaves and Skinner (2010) studied the Royal Forest of Dean in the United Kingdom and concluded that the tourist revisit rate is affected by the destination image [3].

After 1990, the image of tourist destinations was regarded as the research object by Chinese scholars. With the deepening of research, the research object gradually changed from the object of tourist destination to the subject of tourist. Gao Jing et al. (2009) used 4 coastal cities in my country as case sites to analyze their perceived image by using online texts [4]. Wen Jiemin et al. (2019) selected Chongqing's online celebrity tourist attractions Hongyadong Scenic Area as the case site, and merged the image of Hongyadong from the perspective of tourists' perception into five categories [5]. Li Wei et al. (2020) took Zhouzhuang as a case site, use commented on Mafengwo and Tongcheng tourism platform as analysis texts, and concluded that tourists mainly present positive emotions [6].

In foreign studies, the perception of tourist destination image has started early, and the results are more significant, the combination of qualitative and quantitative methods is popular; In domestic studies, 
there are more qualitative studies at the beginning, and quantitative studies gradually increase.

\section{ANALYSIS OF PERCEIVED IMAGE OF TANG PARADISE}

The data sources of this study are Ctrip.com and Mafengwo.com. Sample selection of texts published from January 2019 to January 2020 to ensure timeliness; remove reviews with advertising properties to ensure authenticity; eliminate low-quality reviews with too short reviews and high vocabulary repetition rates to ensure scientific. In the end, 161 tourist reviews from Ctrip.com and 74 from the Mafengwo website were selected as sample texts. And ROST Content 6.0, a content mining software designed by Professor Shenyang of Wuhan University, was used.

\subsection{Cognitive Image}

Preprocess the text: remove expressions and spaces in the text; correct typos; change English characters to Chinese characters. After importing ROST Content 6.0 software, adding "Tang Paradise", "Ziyun Tower", "Qujiang" and other characteristic words that are not suitable for word segmentation to the "custom vocabulary", execute the word segmentation task, and get the text separated by spaces. Then add the blank separated text file to the "Chinese Word Frequency Statistics" window, and set the output of the first 100 high frequency words longer than 2 characters, and output the word frequency at the same time.After statistics, the top 100 high-frequency occurrences totaled 1987 times, and later merged and summed up 4 main categories and 11 sub-categories. The statistical table of cognitive image attribute frequency of tourists in Tang Paradise is obtained. As shown in Table 1:

\subsubsection{Location Division}

From the perception of the location of Tang Paradise, the perception of geographic location is higher than that of administrative divisions.

The words "located" and "place" are mainly mentioned in the geographical location, indicating that tourists pay more attention to the location of the scenic spot; the words mentioned in the administrative divisions are "Xi'an", "Qujiang", " "China", etc., shows that Tang Paradise has become a new landmark in Xi'an in the perception of many tourists.

\subsubsection{Tourist Attraction}

Among the four main categories perceived by tourists, "tourism attractions" accounted for the highest proportion, accounting for $42.68 \%$, indicating that tourists have a deeper perception of tourist attractions in Tang Paradise. The proportions of the three sub-categories under this main category are basically the same.

Under the "Natural Resources" sub-category, the perception of tourists is concentrated in the aspects of "Tang Paradise" and "Waters", indicating that the natural scenery in the Datang Furong Garden is highly attractive to tourists; the "Human Resources" sub-category Below, the words "Datang", "cultural area", and "history" appear frequently, indicating that the overall cultural heritage of the scenic spot from the perspective of tourists' perception is profound, and the style of the Tang Dynasty is fully and objectively reproduced; Under "Leisure and Entertainment", tourists have a deeper perception of words such as "performance" and "water curtain movie". For example, a tourist mentions that "the water curtain movie is the grand finale in the process of playing in Tang Paradise, and it is well worth seeing".

\subsubsection{Facilities and Services}

"Facilities and services" occupy the second place among the four main categories. In the subcategory, "tourism infrastructure" has a higher degree of perception, and more tourists mention "maintenance" and "construction".

Based on the text content, May-July 2019 is the peak period for tourists to report that there are maintenance and construction activities in the scenic area. Many tourists' negative emotions originate from this; tourists' perception of tourism services is mainly concentrated on the tour guide and the park ticket agent which have a relatively low level of perception as a whole; for the accommodation conditions, tourists mention relatively little.

\subsubsection{Tourist Behavior}

Under the main category of "tourist behavior", the proportions of the three subcategories are basically the same. In terms of "tourism consumption", tourists expressed their demand for high ticket prices in Tang Paradise; in terms of "tourism time", most tourists gave comments after visiting at night; in terms of "tourism activities", more tourists expressed that the overall design style and atmosphere of the scenic area brought them a very good travel experience.

In order to further find out the association and direction between the terms, the segmented text is imported into ROST Content 6.0 software, the text content is processed by "social network and semantic network analysis", and it is directly linked to NetDraw software to obtain a semantic network relationship diagram. As shown in Figure 1: 


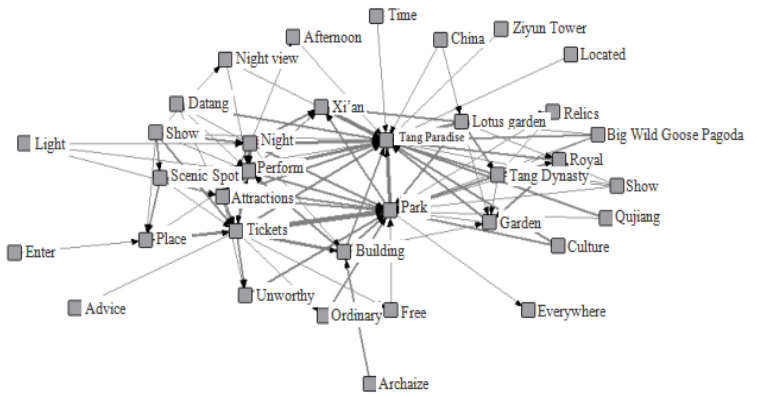

Figure 1 Semantic network analysis diagram of sample text.

According to the semantic network analysis graph, "Tang Paradise", "Park", "Ticket", and "Performance" are important nodes of the entire semantic network analysis graph. At the same time, "Xi'an-Tang Paradise-Tang Dynasty" and "Tang Paradise-Parks-Tickets" and "Night-Performances-Scenic Spots" constitute three important relationship chains in the entire network analysis diagram.

Key words closely related to "Tang Paradise" include "Tang Dynasty", "park", "tickets", etc., reflecting that the Tang Dynasty-like buildings in Tang Paradise have had a huge attraction for tourists; from the perspective of tourists' perception, as a theme park scenic spot, the ticket price of Tang Paradise still needs further discussion; "performance" and "night", "attractions", "night view" and other links, reflect a deep impression on visitors to the site focused on the night and water curtain movie performances.

\subsection{Emotional Image}

Emotional characteristic words express the most intuitive and true feelings of tourists when visiting scenic spots; they are generally divided into three parts: positive emotions, neutral emotions and negative emotions. In this study, we first extract the emotional vocabulary from the sample text, then import it into the ROST Content 6.0 software for the "word segmentation" operation, finally select the "sentiment analysis" function in the software to obtain analysis results, as shown in Table 2:

From the statistical results, the proportions of positive emotions, negative emotions, and neutral emotions are decreasing, respectively, $77.45 \%, 12.76 \%$, and $9.79 \%$. Among the positive emotions, the average intensity accounts for the highest proportion, reaching $33.62 \%$, which shows that the scenic spot has brought tourists a more comfortable and satisfying travel experience; among the negative emotions, the average intensity accounts for the highest proportion, reaching $11.06 \%$, combined with the sample text content, it can be seen that the scenic spot needs to be improved in terms of ticket and infrastructure; neutral emotions accounted for $9.79 \%$, indicating that this scenic spot has reached a state of "no dissatisfaction" in most tourists.

After that, the emotional vocabulary that has been extracted from the text is preprocessed, such as merging "very pretty" and "very beautiful" into "very beautiful", and convert some English words into corresponding Chinese words. Import it into the ROST Content 6.0 software, use the "Chinese word frequency statistics" function to analyze, derive the top 20 emotional-related feature words, and determine their emotional attributes, which are divided into three categories: positive, negative and neutral, then the emotional image of the scenic spot can be obtained. As shown in Table 3:

Table 1. Frequency statistics of tourists' cognitive image attributes in Tang Paradise

\begin{tabular}{|c|c|c|c|c|c|}
\hline Main category & Frequency & Percentage & Subcategory & Frequency & Percentage \\
\hline \multirow{2}{*}{ Location division } & \multirow{2}{*}{168} & \multirow{2}{*}{$8.45 \%$} & Location & 103 & $5.18 \%$ \\
\hline & & & Administrative divisions & 65 & $3.27 \%$ \\
\hline \multirow{3}{*}{ Tourist attraction } & \multirow{3}{*}{848} & \multirow{3}{*}{$42.68 \%$} & Natural resources & 249 & $12.53 \%$ \\
\hline & & & Human resources & 372 & $18.72 \%$ \\
\hline & & & Leisure and entertainment & 227 & $11.42 \%$ \\
\hline \multirow{3}{*}{ Facilities and services } & \multirow{3}{*}{498} & \multirow{3}{*}{$25.06 \%$} & Tourism infrastructure & 300 & $15.10 \%$ \\
\hline & & & Travel services & 179 & $9.01 \%$ \\
\hline & & & Accommodation conditions & 19 & $0.96 \%$ \\
\hline \multirow{3}{*}{ Tourist behavior } & \multirow{3}{*}{473} & \multirow{3}{*}{$23.80 \%$} & Tourism consumption & 148 & $7.45 \%$ \\
\hline & & & Travel time & 197 & $9.91 \%$ \\
\hline & & & Travel Activity & 128 & $6.44 \%$ \\
\hline
\end{tabular}


Table 2. Sentiment analysis statistical results of sample text

\begin{tabular}{|c|c|c|l|c|}
\hline Mood category & Quantity & Proportion & Strength & Proportion \\
\hline Positive emotions & 182 & $77.45 \%$ & General & $33.62 \%$ \\
\cline { 3 - 5 } & & & Moderate & $21.70 \%$ \\
\cline { 3 - 5 } & & & Height & $22.13 \%$ \\
\hline Neutral mood & 23 & $9.79 \%$ & $/$ & $9.79 \%$ \\
\hline Negative emotions & 30 & $12.76 \%$ & General & $11.06 \%$ \\
\cline { 4 - 5 } & & & Moderate & $1.70 \%$ \\
\cline { 4 - 5 } & & & Height & $0.00 \%$ \\
\hline Total & 235 & $100.00 \%$ & & $100.00 \%$ \\
\hline
\end{tabular}

Table 3. Top 20 feature words of sentiment vocabulary in sample text

\begin{tabular}{|c|c|c|c|c|c|}
\hline Serial number & Emotional vocabulary & Emotional attributes Serial number & Emotional vocabulary & Emotional attributes \\
\hline 1 & Very beautiful & positive & 11 & Features & positive \\
\hline 2 & expensive & negative & 12 & New & neutral \\
\hline 3 & good looking & positive & 13 & Resplendent & positive \\
\hline 4 & ordinary & neutral & 14 & lively & positive \\
\hline 5 & worth it & positive & 15 & Attentive & positive \\
\hline 6 & tired & negative & 16 & atmosphere & positive \\
\hline 7 & Very big & positive & 17 & Pretty & positive \\
\hline 8 & style & positive & 18 & wonderful & positive \\
\hline 9 & Most beautiful & positive & 19 & Bright & positive \\
\hline 10 & Beautiful & positive & 20 & Convenience & positive \\
\hline
\end{tabular}

\subsubsection{Positive Perception}

After summarizing, the top 20 emotional positive words appear 60 times, accounting for $76.92 \%$, and the words involved include "beautiful", "good-looking", "brilliant" and so on. Based on the "cognition-emotion" model, it can be known that the specific things, facilities, and services in the scenic area directly affect the formation of tourists' cognitive image of the scenic area. On this basis, the emotions of the tourists will correspond performance and changes. For example, during a visit to the scenic spot, some tourists mentioned that the staff in the scenic spot smiled when giving directions for them, and then the emotional word "attentive" appeared in their comments, showing their positive emotional experience.

\subsubsection{Negative Perception}

After summarizing, the top 20 emotional negative words appeared 10 times, accounting for $12.82 \%$, and the words involved included "expensive" and "tired". Based on the "cognitive-emotional" model, tourists visiting Tang Paradise have more emotional expressions that are "expensive" when they come into contact with tickets. For example, some tourists mentioned that some scenic spots could not be visited normally due to the maintenance of the scenic spots, but the ticket fees received did not decrease.For "tired", what is more combined is the category of "tourist behavior" in the image perceived by tourists. Some tourists mentioned that because they did not follow a reasonable route to visit in the scenic spots, the overall visit time was longer, so produce the emotion of "tired". In a word, tourists have more negative perceptions of "tourism infrastructure" and "tourism consumption".

\section{IN CONCLUSION}

Based on the above of Tang Paradise of cognitive image and affective image analysis, which can be concluded that Tang Paradise in tourists under the perspective of the overall image: Tang Paradise is a natural, cultural resources rich scenic spot, is a with tang has turned into the theme of architecture design style characteristic scenic area, is a suitable for the night tour performances and varied. At the same time, it is also a scenic spot that needs to be improved in terms of tourism consumption and tourism infrastructure. The promotion of the tourism image of Tang Paradise can be started from four aspects: strengthening the construction of tourism infrastructure, digging into the cultural 
connotation of the scenic spot, improving the quality of tourism service of the scenic spot and improving the feedback mechanism of tourists after their tour.

\section{REFERENCES}

[1] Hunt J D.Image-Afactor in Tourism [D].1971.

[2] Choi S,Lehtoa X Y,Morrison A M.Destination image representation on the web: Content analysis of Macau travel related websites[J].Tourism Management,2007,28(1) : 118-129.

[3] Greaves N,Skinner H.The importance of destination image analysis to UK rural tourism[J].Marketing Intelligence \& Planning,2010,28( 4) : 486-507.

[4] Gao Jing, Zhang Yonggang, Zhuang Dongquan. Research on Domestic Tourists' Perceived Image of Coastal Tourist Cities-Based on Text Analysis of Ctrip and Tongcheng Net Netizens' Comments [J]. Consumer Economy, 2009(3): 6265.(In Chinese)

[5] Wen Jiemin, Yu Ying, Liu Xuewei, Liu Xuemin, Shi Pengfei. Research on the image perception of "net celebrity" tourist destinations based on network text analysis_-A case study of Chongqing Hongyadong Scenic Area[J]. Tourism Research, 2019,11(02):44-57.(In Chinese)

[6] Li Wei, Li Duo. Research on the Image Perception and Tourist Evaluation of Featured Tourist Destinations Based on Domestic Travel Notes_-Taking Zhouzhuang, an ancient water town in Suzhou as an example [J]. Statistics and Management, 2020, 35(01): 115- 120.(In Chinese) 